Proceedings of the Edinburgh Mathematical Society (2002) 45, 653-671 (C)

DOI:10.1017/S001309150000119X Printed in the United Kingdom

\title{
CONTINUED FRACTIONS WITH BOUNDED PARTIAL QUOTIENTS
}

\author{
J. L. DAVISON \\ Department of Mathematics and Computer Science, Laurentian University, \\ Sudbury, Ontario, Canada P3E 2C6 (les@cs.laurentian.ca)
}

(Received 28 November 2000)

Abstract Precise bounds are given for the quantity

$$
L(\alpha)=\frac{\limsup _{m \rightarrow \infty}(1 / m) \ln q_{m}}{\liminf \inf _{m \rightarrow \infty}(1 / m) \ln q_{m}},
$$

where $\left(q_{m}\right)$ is the classical sequence of denominators of convergents to the continued fraction $\alpha=$ $\left[0, u_{1}, u_{2}, \ldots\right]$ and $\left(u_{m}\right)$ is assumed bounded, with a distribution.

If the infinite word $\boldsymbol{u}=u_{1} u_{2} \ldots$ has arbitrarily large instances of segment repetition at or near the beginning of the word, then we quantify this property by means of a number $\gamma$, called the segmentrepetition factor.

If $\alpha$ is not a quadratic irrational, then we produce a specific sequence of quadratic irrational approximations to $\alpha$, the rate of convergence given in terms of $L$ and $\gamma$. As an application, we demonstrate the transcendence of some continued fractions, a typical one being of the form $\left[0, u_{1}, u_{2}, \ldots\right]$ with $u_{m}=1+\lfloor m \theta\rfloor \bmod n, n \geqslant 2$, and $\theta$ an irrational number which satisfies any of a given set of conditions.

Keywords: transcendence; approximation; distribution

AMS 2000 Mathematics subject classification: Primary 11A55

Secondary 11B37

\section{Introduction}

Suppose $\Sigma$ is a finite set of positive integers. If $\left(u_{m}\right)_{m \geqslant 1}$ is an infinite sequence with $u_{m} \in \Sigma$ for each $m \geqslant 1$, then we let $\boldsymbol{u}$ be the infinite word $u_{1} u_{2} \ldots$ and say that $\boldsymbol{u}$ takes its values from $\Sigma$. Suppose the continued fraction $\alpha=\left[0, u_{1}, u_{2}, \ldots\right]$ has convergents

$$
\left(\frac{p_{m}}{q_{m}}\right)_{m \geqslant 0}
$$

Then we define

$$
L(\alpha)=\frac{\limsup _{m \rightarrow \infty}(1 / m) \ln q_{m}}{\liminf \inf _{m \rightarrow \infty}(1 / m) \ln q_{m}}
$$

and set $L(\boldsymbol{u})=L(\alpha)$. 
It is evident that $L(\alpha) \geqslant 1$ and that $L(\alpha)=1$ if and only if $\lim _{m \rightarrow \infty}(1 / m) \ln q_{m}$ exists. It is of interest to be able to provide precise upper estimates for $L(\alpha)$. Of course, if we only know that $a \leqslant u_{m} \leqslant b$ for all $m \geqslant 1$, then it is easy to see that

$$
L(\alpha) \leqslant \ln \left(\frac{1}{2}\left(b+\sqrt{b^{2}+4}\right)\right) / \ln \left(\frac{1}{2}\left(a+\sqrt{a^{2}+4}\right)\right)
$$

and that this bound can be attained.

If we assume that $|\Sigma|=2$ and that $\boldsymbol{u}$ takes each of the two values with a frequency (see Definition 3.1), then the authors of [1] have shown that $L(\boldsymbol{u})<1.13$, irrespective of the particular elements of $\Sigma$. In $\S \S 2$ and 3 , we extend this result to any finite set, on the assumption that $\boldsymbol{u}$ takes each of the values in $\Sigma$ with a frequency. In $\S 4$ we give a more precise estimate, provided that $\boldsymbol{u}$ is uniformly distributed (that is, each value is taken with the same frequency).

Our main goal in this paper is to prove transcendence of a certain family of continued fractions. For this, we also need to consider the property that the infinite word $\boldsymbol{u}$ has arbitrarily large instances of segment repetition near the beginning of $\boldsymbol{u}$. A special case of this concept was discussed in [1]. More formally we make the following definition.

Definition 1.1. Suppose $\gamma \in \mathbb{R}$ with $\gamma \geqslant 1$. The infinite word $\boldsymbol{w}=w_{1} w_{2} w_{3} \ldots$ is said to have a segment expansion factor greater than or equal to $\gamma$ if there exist three infinite sequences of finite words $\left\{U_{k}\right\}_{k \geqslant 1},\left\{V_{k}\right\}_{k \geqslant 1},\left\{W_{k}\right\}_{k \geqslant 1}$ which satisfy all the following conditions.

(1) $U_{k} V_{k} W_{k}$ is a prefix of $\boldsymbol{w}$.

(2) $\lim _{k \rightarrow \infty}\left|V_{k}\right|=\infty$, where $\left|V_{k}\right|$ is the length of $V_{k}$.

(3) $W_{k}$ is a prefix of $V_{k}^{s}$ for some positive integer $s$.

(4) $\liminf _{k \rightarrow \infty} \frac{\left|U_{k} V_{k} W_{k}\right|}{\left|U_{k}\right|+\left|U_{k} V_{k}\right|}=\gamma$.

Finally, we will say $\boldsymbol{w}$ has a prefix expansion factor greater than or equal to $\gamma$ if we can take $U_{k}=\lambda$ for all $k \geqslant 1$.

In $\S 5$ we provide explicit computations of the segment expansion factor for the infinite word $\boldsymbol{w}=w_{1} w_{2} \ldots$, where $w_{m}=\lfloor m \theta\rfloor \bmod n$ and $\theta$ is an irrational with $0<\theta<1$.

In the final section of the paper we first prove that if $\alpha$ is not a quadratic irrational (that is, $\boldsymbol{u}$ is not ultimately periodic) and if $\boldsymbol{u}$ has a segment expansion factor greater than or equal to $\gamma$, then there is a sequence of quadratic irrationals $\left(\alpha_{k}\right)$ which satisfy

$$
\left|\alpha-\alpha_{k}\right|<\frac{1}{H\left(\alpha_{k}\right)^{2 \gamma / L(\alpha)}},
$$

where $H\left(\alpha_{k}\right)$ denotes the height of $\alpha_{k}$.

With the aid of Schmidt's Theorem [9], we then obtain a transcendence result for a special class of continued fractions derived from the words studied in $\S 5$. 


\section{Basic terminology and the trace inequality}

Let $\Sigma=\left\{a_{1}, a_{2}, \ldots, a_{n}\right\}$ be a finite set of $n \geqslant 2$ positive integers, ordered so that $1 \leqslant a_{1}<a_{2}<\cdots<a_{n}$. Let $\boldsymbol{u}=\left(u_{m}\right)_{m \geqslant 1} \in \Sigma^{\mathbb{N}}$ be any infinite sequence with values in $\Sigma$.

Consider the sequence $\left(q_{m}\right)_{m \geqslant-1}$ defined by

$$
q_{-1}=0, \quad q_{0}=1, \quad q_{m}=u_{m} q_{m-1}+q_{m-2} \quad \text { for } m \geqslant 1 .
$$

The sequence $\left(q_{m}\right)_{m \geqslant-1}$ so defined is the sequence of denominators of the convergents to the continued fraction $\left[0, u_{1}, u_{2}, \ldots\right]$. Readers can consult $[\mathbf{6}]$ or $[\mathbf{8}]$ for information on standard continued fraction theory. We will say $\boldsymbol{u}$ generates the sequence $\left(q_{m}\right)$. The statement (2.1) can be expressed in matrix form by

$$
\left[\begin{array}{c}
q_{0} \\
q_{-1}
\end{array}\right]=\left[\begin{array}{l}
1 \\
0
\end{array}\right], \quad\left[\begin{array}{c}
q_{m} \\
q_{m-1}
\end{array}\right]=\left[\begin{array}{cc}
u_{m} & 1 \\
1 & 0
\end{array}\right]\left[\begin{array}{l}
q_{m-1} \\
q_{m-2}
\end{array}\right] \quad \text { for } m \geqslant 1 .
$$

If we write

$$
A_{i}=\left[\begin{array}{cc}
a_{i} & 1 \\
1 & 0
\end{array}\right] \quad \text { for } 1 \leqslant i \leqslant n
$$

then it can be shown that the semigroup $S_{n}=S\left(A_{1}, A_{2}, \ldots, A_{n}\right)$ generated by the matrices $A_{1}, A_{2}, \ldots, A_{n}$ is free, so we can identify the matrices in $S_{n}$ with the corresponding words (strings) in the symbols $A_{1}, A_{2}, \ldots, A_{n}$. The length of such a word $W$, denoted by $|W|$, is the number of symbols (counting repetitions) that occur in $W$. If $\mathcal{S}_{n}^{-}$denotes the set of those matrices in $S_{n}$ with determinant equal to -1 , then $W \in \mathcal{S}_{n}^{-}$if and only if $|W|$ is odd. The trace of $W$, denoted by $\operatorname{tr}(W)$, is the trace of the matrix $W$.

We can write the preceding matrix recurrence in the form

$$
\left[\begin{array}{c}
q_{m} \\
q_{m-1}
\end{array}\right]=W_{m}\left[\begin{array}{l}
1 \\
0
\end{array}\right]
$$

where $W_{m}\left(A_{1}, A_{2}, \ldots, A_{n}\right)$ is a word of length $m$ in the matrices $A_{1}, A_{2}, \ldots, A_{n}$. We will say that $W_{m}$ is associated with $q_{m}$.

If $\rho(M)$ denotes the spectral radius of the real square matrix $M$, then the $L^{2}$-norm of $M$, written as $\|M\|$, equals $\sqrt{\rho\left(M^{\mathrm{t}} M\right)}$. In particular, $\left\|A_{i}\right\|=\rho\left(A_{i}\right)=\frac{1}{2}\left(a_{i}+\sqrt{a_{i}^{2}+4}\right)$.

The first result, proved in [1] , shows the connection between $q_{m}$ and $W_{m}$.

Proposition 2.1. The following inequalities hold:

(a) $q_{m} \leqslant\left\|W_{m}\right\| ;$ and

(b) $q_{m} \geqslant \frac{1}{2} \operatorname{tr} W_{m}$.

In order to proceed further, it is thus essential to consider the trace of words in $\mathcal{S}_{n}$. As much of the first part is easily derivable from the $n=2$ case described in some detail in $[\mathbf{1}]$, we will be brief in our exposition. 
If

$$
X=\left[\begin{array}{ll}
\alpha & \beta \\
\gamma & \delta
\end{array}\right] \in \mathcal{S}_{n},
$$

let $\Phi_{X}$ be the map $x \rightarrow((\delta x+\gamma) /(\beta x+\alpha))$. Then $\Phi_{M N}=\Phi_{M} \circ \Phi_{N}$ and $\Phi_{X}$ has two fixed points $x_{X}, y_{X}$ with $x_{X}<y_{X}$. For $1 \leqslant i, j \leqslant n$ put

$x_{i j}=x_{A_{i} A_{j}}=\frac{1}{2}\left(-a_{j}-\sqrt{a_{j}^{2}+4 a_{j} / a_{i}}\right) \quad$ and $\quad y_{i j}=y_{A_{i} A_{j}}=\frac{1}{2}\left(-a_{j}+\sqrt{a_{j}^{2}+4 a_{j} / a_{i}}\right)$.

Note that $x_{i i}=x_{A_{i}}$ and $y_{i i}=y_{A_{i}}$ for $1 \leqslant i \leqslant n$.

Lemma 2.2. For $1 \leqslant i, j \leqslant n$ the following hold:

(a) $\left(1 /\left(x_{i j}+a_{j}\right)\right)=x_{j i}$;

(b) $y_{i j} x_{j i}=-1$; and

(c) $x_{i j}>-\left(a_{j}+\left(1 / a_{i}\right)\right)$.

Lemma 2.3. The fixed points $\left(x_{i j}\right),\left(y_{i j}\right)$ are totally ordered as follows:

(a) $-a_{j}-1<x_{1 j}<x_{2 j}<\cdots<x_{n j}<-a_{j}, 1 \leqslant j \leqslant n$; and

(b) $\left(1 /\left(a_{i}+1\right)\right)<y_{i 1}<y_{i 2}<\cdots<y_{i n}<\left(1 / a_{i}\right), 1 \leqslant i \leqslant n$.

The proofs of these three lemmas are omitted.

Proposition 2.4. We have

$$
\operatorname{tr}\left(A_{1} A_{n} X\right) \geqslant \rho\left(A_{1} A_{n}\right) \operatorname{tr}(X) \quad \text { for any } X \in \mathcal{S}_{n}^{-}\left(A_{1}, A_{2}, \ldots, A_{n}\right) .
$$

Proof. As in [1] , it suffices to show that (i) $\beta x_{1 n}+\alpha<0$, and (ii) $x_{n 1} \leqslant \Phi_{X}\left(x_{1 n}\right) \leqslant$ $y_{1 n}$, where

$$
X=\left[\begin{array}{ll}
\alpha & \beta \\
\gamma & \delta
\end{array}\right] .
$$

These two statements are proved by induction on the (odd) length of $X$. Lemmas 2.2 and 2.3 give the basis case $(|X|=1)$ and, if we let $\mathcal{U}_{n}=\left\{A_{i} A_{j} ; 1 \leqslant i, j \leqslant n\right\}$, the inductive step hinges on the fact that

$$
\min \left\{\frac{m_{1}}{m_{2}}: M=\left[\begin{array}{ll}
m_{1} & m_{2} \\
m_{3} & m_{4}
\end{array}\right] \in \mathcal{U}_{n}\right\}=a_{1}+\frac{1}{a_{n}} .
$$

The details are left to the reader.

So far the extension to more than two arguments is fairly direct. We now come to the proposition that allows us to take out an $A_{1} A_{n}$ term when the word does not have an adjacent pair $A_{1}, A_{n}$. 
Proposition 2.5. Suppose $n \geqslant 3$, and let $W^{\mathrm{R}}$ denote the transposed (or reverse) string of $W$. Then $\operatorname{tr}\left(A_{1} W A_{n} X\right) \geqslant \operatorname{tr}\left(A_{1} A_{n} W^{\mathrm{R}} X\right)$ for all $W \in \mathcal{S}\left(A_{2}, \ldots, A_{n-1}\right)$ and for all $X \in \mathcal{S}\left(A_{1}, A_{2}, \ldots, A_{n}\right)$ that do not start with $A_{1}$.

Proof. Let

$$
W=\left[\begin{array}{ll}
u & v \\
w & x
\end{array}\right] .
$$

Then (noting that $a_{2} \geqslant 2$ ) it is easy to establish by induction that

$$
w<u<a_{n} w+x
$$

Also

$$
W A_{n}-A_{n} W^{\mathrm{R}}=\left[\begin{array}{cc}
0 & u-a_{n} w-x \\
a_{n} w+x-u & 0
\end{array}\right],
$$

which by (2.3) is of the form

$$
\left[\begin{array}{cc}
0 & -y_{n} \\
y_{n} & 0
\end{array}\right]
$$

with $y_{n}>0$.

Since $\operatorname{tr}\left(A_{1}\left(W A_{n}-A_{n} W^{\mathrm{R}}\right) X\right)=y_{n}\left(\alpha-a_{1} \gamma-\delta\right)$, it suffices to show that $\alpha \geqslant a_{1} \gamma+\delta$ for all $X$ not starting with $A_{1}$. If $X=A_{i}$ for some $i, 2 \leqslant i \leqslant n$, then $a_{i} \geqslant a_{1}+1$, so the result holds in this case. If $|X| \geqslant 2$, then we can write $X=A_{i} U A_{j}$, where $2 \leqslant i \leqslant n$, $1 \leqslant j \leqslant n$, and $U \in \mathcal{S}_{n} \cup\{I\}$. It is easy to see that $\alpha \geqslant a_{1} \gamma+\delta$ in this situation.

\section{Infinite words with frequency}

As mentioned in $\S 1$, we must impose some condition on $\boldsymbol{u}=\left(u_{m}\right)_{m \geqslant 1}$ in order to expect a better estimate for $L(\boldsymbol{u})$. It turns out that a natural condition to impose is that each $a_{i} \in \Sigma$ occurs in $\boldsymbol{u}$ with a frequency $\alpha_{i}$. Specifically, we make the following definition.

Definition 3.1. For $1 \leqslant i \leqslant n$ put $\alpha_{i}(m)=\left|\left\{1 \leqslant k \leqslant m: u_{k}=a_{i}\right\}\right|$. If for each $i$, $\lim _{m \rightarrow \infty}\left(\alpha_{i}(m) / m\right)$ exists, say equal to $\alpha_{i}$, then we say that $\boldsymbol{u}$ is a word with frequency

$$
\boldsymbol{\alpha}=\left(\alpha_{1}, \alpha_{2}, \ldots, \alpha_{n}\right) .
$$

Let us write $\boldsymbol{A}=\left(A_{1}, A_{2}, \ldots, A_{n}\right)$, where, as usual, $A_{i}$ denotes the matrix

$$
\left[\begin{array}{cc}
a_{i} & 1 \\
1 & 0
\end{array}\right]
$$

Furthermore, put $M(\boldsymbol{A}, \boldsymbol{\alpha})=\sum_{i=1}^{n} \alpha_{i} \ln \rho\left(A_{i}\right)$. Then we have the following proposition (cf. $[\mathbf{1}, \mathbf{3}])$.

Proposition 3.2. Suppose $\boldsymbol{u}$ is an infinite word with values taken from $\Sigma$ with frequency $\boldsymbol{\alpha}$ and suppose $\boldsymbol{u}$ generates $\left(q_{m}\right)$. Then $\lim \sup _{m \rightarrow \infty}(1 / m) \ln q_{m} \leqslant M(\boldsymbol{A}, \boldsymbol{\alpha})$. 
Proof. Using our previously introduced notation we have

$$
\left\|W_{m}\right\| \leqslant \rho\left(A_{1}\right)^{\alpha_{1}(m)} \rho\left(A_{2}\right)^{\alpha_{2}(m)} \ldots \rho\left(A_{n}\right)^{\alpha_{n}(m)} .
$$

By Proposition 2.1 it follows that

$$
\limsup _{m \rightarrow \infty}\left(\frac{1}{m}\right) \ln q_{m} \leqslant \limsup _{m \rightarrow \infty} \frac{\ln \left\|W_{m}\right\|}{m} \leqslant \sum_{i=1}^{n} \alpha_{i} \ln \rho\left(A_{i}\right)=M(\boldsymbol{A}, \boldsymbol{\alpha}) .
$$

The task ahead will be to construct a piecewise linear (in $\boldsymbol{\alpha}$ ) function $H(\boldsymbol{A}, \boldsymbol{\alpha})$ that satisfies the inequality $\lim \inf _{m \rightarrow \infty}(1 / m) \ln q_{m} \geqslant H(\boldsymbol{A}, \boldsymbol{\alpha})$.

First we note the following lemma from Proposition 2.1.

Lemma 3.3. $\liminf _{m \rightarrow \infty}\left(\frac{1}{m}\right) \ln q_{m} \geqslant \liminf _{\substack{m \rightarrow \infty \\ m \text { odd }}}\left(\frac{1}{m}\right) \ln \operatorname{tr} W_{m}$.

Proof. $\left(q_{m}\right)$ is an increasing sequence so that

$$
\liminf _{m \rightarrow \infty}\left(\frac{1}{m}\right) \ln q_{m}=\liminf _{\substack{m \rightarrow \infty \\ m \text { odd }}}\left(\frac{1}{m}\right) \ln q_{m} \geqslant \liminf _{\substack{m \rightarrow \infty \\ m \text { odd }}}\left(\frac{1}{m}\right) \ln \operatorname{tr} W_{m} .
$$

We now use Proposition 2.4 and Proposition 2.5 to get the following theorem.

Theorem 3.4. Let $W_{m}\left(A_{1}, A_{2}, \ldots, A_{n}\right)$ be a word of odd length $m$.

(a) If $\alpha_{1}(m) \leqslant \alpha_{n}(m)$, then

$$
\operatorname{tr} W_{m}\left(A_{1}, A_{2}, \ldots, A_{n}\right) \geqslant \rho\left(A_{1} A_{n}\right)^{\alpha_{1}(m)} \operatorname{tr} W_{m-2 \alpha_{1}(m)}\left(A_{2}, A_{3}, \ldots, A_{n}\right),
$$

where the number of occurrences of $A_{i}$ in $W_{m-2 \alpha_{1}(m)}$ is $\alpha_{i}(m)$ for $2 \leqslant i \leqslant n-1$, and $\alpha_{n}(m)-\alpha_{1}(m)$ for $i=n$.

(b) If $\alpha_{n}(m) \leqslant \alpha_{1}(m)$, then

$$
\operatorname{tr} W_{m}\left(A_{1}, A_{2}, \ldots, A_{n}\right) \geqslant \rho\left(A_{1} A_{n}\right)^{\alpha_{n}(m)} \operatorname{tr} W_{m-2 \alpha_{n}(m)}\left(A_{1}, A_{2}, \ldots, A_{n-1}\right),
$$

where the number of occurrences of $A_{i}$ in $W_{m-2 \alpha_{n}(m)}$ is $\alpha_{1}(m)-\alpha_{n}(m)$ for $i=1$ and $\alpha_{i}(m)$ for $2 \leqslant i \leqslant n-1$.

Proof. If $W_{m}$ has an adjacent $A_{1} A_{n}$ or $A_{n} A_{1}$, we use Proposition 2.4, since tr is invariant under cyclic permutation and under transpose (or reverse), to obtain

$$
\operatorname{tr} W_{m} \geqslant \rho\left(A_{1} A_{n}\right) \operatorname{tr} W_{m-2}\left(A_{1}, \ldots, A_{n}\right) .
$$

We can continue to remove adjacent $A_{1} A_{n}$ in such a manner until no adjacency remains. At that point we can use Proposition 2.5 instead, which will produce an adjacency between $A_{1}, A_{n}$ for which Proposition 2.4 will again be used. Going back and forth in this manner, we will either exhaust the $A_{1} \mathrm{~s}$ first $\left(\alpha_{1}(m)<\alpha_{n}(m)\right)$ or the $A_{n} \mathrm{~s}$ $\left(\alpha_{n}(m)<\alpha_{1}(m)\right)$ or possibly exhaust them together $\left(\alpha_{1}(m)=\alpha_{n}(m)\right)$. The desired inequalities are now clear. 
The effect of Theorem 3.4 is to reduce the number of variables in the argument by one, or possibly two. Consider for example a word $\boldsymbol{u}$ with frequency $\boldsymbol{\alpha}$, where $\alpha_{1}<\alpha_{n}$. Then $\alpha_{1}(m)<\alpha_{n}(m)$ for all $m \geqslant m_{0}$, say, and also $m^{*}=m-2 \alpha_{1}(m) \rightarrow \infty$, since $\alpha_{1}<\frac{1}{2}$. Thus

$$
\liminf _{\substack{m \rightarrow \infty \\ m \text { odd }}}\left(\frac{1}{m}\right) \ln \operatorname{tr} W_{m} \geqslant \alpha_{1} \ln \rho\left(A_{1} A_{n}\right)+\left(1-2 \alpha_{1}\right) \liminf _{\substack{m^{*} \rightarrow \infty \\ m^{*} \rightarrow \text { odd }}}\left(\frac{1}{m^{*}}\right) \ln \operatorname{tr} W_{m^{*}},
$$

and the frequencies of $A_{i}$ in $W_{m^{*}}$ are $\alpha_{i} /\left(1-2 \alpha_{1}\right)$ for $2 \leqslant i \leqslant n-1$ and $\left(\alpha_{n}-\alpha_{1}\right) /\left(1-2 \alpha_{1}\right)$ for $i=n$.

The right-hand side of equation (3.1) has the makings of part of the recursive definition of $H(\boldsymbol{A}, \boldsymbol{\alpha})$, but we must be careful about the relationship between the different $\boldsymbol{\alpha}$ s. We therefore consider the various domains of definition of the proposed $H(\boldsymbol{A}, \boldsymbol{\alpha})$.

For $n \geqslant 1$ let us put

$$
D_{n}=\left\{\boldsymbol{\alpha} \in R^{n}: \alpha_{i} \geqslant 0 \text { for } 1 \leqslant i \leqslant n \text { and } \sum_{i=1}^{n} \alpha_{i}=1\right\} .
$$

Note, in particular, that $D_{1}=\{1\}$. For $n \geqslant 2$ we also need the following special points in $D_{n}$ :

(a) for $1 \leqslant i \leqslant n$, put $\boldsymbol{e}_{i}=\left(\alpha_{k}\right)$, where $\alpha_{k}=1$ if $k=i$ and 0 otherwise; and

(b) for $1 \leqslant i<j \leqslant n$, put $\boldsymbol{f}_{i j}=\frac{1}{2}\left(\boldsymbol{e}_{i}+\boldsymbol{e}_{j}\right)$.

We are going to break up the set $D_{n}$ into $2^{n-1}$ subdomains, which we can usefully parametrize using binary strings of length $(n-1)$.

\section{Definition 3.5.}

(a) If $\lambda$ denotes the empty string, put $\Delta_{\lambda}=\{1\}$.

(b) Let $n \geqslant 2$ and suppose $\Delta_{\boldsymbol{b}}$ has been defined for all binary strings $\boldsymbol{b}$ of length $(n-2)$. Then we set

$$
\Delta_{1 \boldsymbol{b}}=\left\{\boldsymbol{\alpha} \in D_{n}: \alpha_{1} \leqslant \alpha_{n} \text { and }\left(\alpha_{2}, \alpha_{3}, \ldots, \alpha_{n-1}, \alpha_{n}-\alpha_{1}\right) \in\left(1-2 \alpha_{1}\right) \Delta_{\boldsymbol{b}}\right\}
$$

and

$$
\Delta_{0 \boldsymbol{b}}=\left\{\boldsymbol{\alpha} \in D_{n}: \alpha_{n} \leqslant \alpha_{1} \text { and }\left(\alpha_{1}-\alpha_{n}, \alpha_{2}, \ldots, \alpha_{n-1}\right) \in\left(1-2 \alpha_{n}\right) \Delta_{\boldsymbol{b}}\right\} .
$$

Proposition 3.6. Let $n \geqslant 1$. Then

(a) $D_{n}=\bigcup \Delta_{\boldsymbol{b}}$, taken over all binary strings $\boldsymbol{b}$ of length $(n-1)$; and

(b) if $\boldsymbol{b}$ is a binary string of length $(n-1)$, then $\Delta_{\boldsymbol{b}}$ is a simplex with vertices in the set $\left\{\boldsymbol{f}_{i j}: 1 \leqslant i<j \leqslant n\right\} \bigcup\left\{\boldsymbol{e}_{i}: 1 \leqslant i \leqslant n\right\}$. 
Proof. Both parts are proved by induction.

Note that if $\alpha \in \Delta_{1 b}$, then we can write

$$
\boldsymbol{\alpha}=2 \alpha_{1} \boldsymbol{f}_{1 n}+\left(1-2 \alpha_{1}\right)(0, \boldsymbol{\beta}) \quad \text { for some } \boldsymbol{\beta} \in \Delta_{\boldsymbol{b}},
$$

and if $\alpha \in \Delta_{0 b}$, then we have

$$
\boldsymbol{\alpha}=2 \alpha_{n} \boldsymbol{f}_{1 n}+\left(1-2 \alpha_{n}\right)(\boldsymbol{\beta}, 0) \text { for some } \boldsymbol{\beta} \in \Delta_{\boldsymbol{b}} .
$$

These decompositions are unique if $\alpha_{1}<\frac{1}{2}$ in (3.2) and $\alpha_{n}<\frac{1}{2}$ in (3.3). We are now ready to define $H$ recursively on $D_{n}$.

\section{Definition 3.7.}

(a) If $n=1$, then set $H(A, 1)=\ln \rho(A)$, for $A$ a matrix of the form

$$
\left[\begin{array}{ll}
a & 1 \\
1 & 0
\end{array}\right]
$$

with $a$ a positive integer.

(b) Suppose $n \geqslant 2$ and assume $H(\boldsymbol{A}, \boldsymbol{\alpha})$ has been defined for $\boldsymbol{\alpha} \in D_{n-1}$. If $\boldsymbol{\alpha} \in D_{n}$, then by Proposition 3.6 either $\boldsymbol{\alpha} \in \Delta_{1 \boldsymbol{b}}$ or $\boldsymbol{\alpha} \in \Delta_{0 \boldsymbol{b}}$ for some $\boldsymbol{b}$ of length $n-2$.

If $\boldsymbol{\alpha} \in \Delta_{1 \boldsymbol{b}}$, then in light of (3.2) we set

$$
H(\boldsymbol{A}, \boldsymbol{\alpha})=\alpha_{1} \ln \rho\left(A_{1} A_{n}\right)+\left(1-2 \alpha_{1}\right) H(\boldsymbol{B}, \boldsymbol{\beta}),
$$

where $\boldsymbol{B}=\left(A_{2}, A_{3}, \ldots, A_{n}\right)$.

If $\boldsymbol{\alpha} \in \Delta_{0 \boldsymbol{b}}$, then using (3.3) we set

$$
H(\boldsymbol{A}, \boldsymbol{\alpha})=\alpha_{n} \ln \rho\left(A_{1} A_{n}\right)+\left(1-2 \alpha_{n}\right) H(\boldsymbol{C}, \boldsymbol{\beta}) \quad \text { where } \boldsymbol{C}=\left(A_{1}, A_{2}, \ldots, A_{n-1}\right) .
$$

Proposition 3.8. Let $n \geqslant 1$. Then

(a) $H$ is a well-defined function on $D_{n}$; and

(b) $H$ is linear in $\boldsymbol{\alpha}$ on $\Delta_{\boldsymbol{b}}$ for any $\boldsymbol{b}$. In other words, $H$ is piecewise linear in $\boldsymbol{\alpha}$ on $D_{n}$.

\section{Proof.}

(a) Easily checked (by induction).

(b) If, for example, $\boldsymbol{\alpha} \in \Delta_{1 \boldsymbol{b}}$ and $\alpha_{1}<\frac{1}{2}$, then (3.2) gives

$$
\boldsymbol{\beta}=\frac{1}{1-2 \alpha_{1}}\left(\alpha_{2}, \alpha_{3}, \ldots, \alpha_{n-1}, \alpha_{n}-\alpha_{1}\right) .
$$

If $H(\boldsymbol{B}, \boldsymbol{\beta})$ is linear in $\boldsymbol{\beta}$ on $\Delta_{\boldsymbol{b}}$, then $H(\boldsymbol{A}, \boldsymbol{\alpha})$ is linear in $\boldsymbol{\alpha}$ on $\Delta_{1 \boldsymbol{b}}$. 
If we set $n=2$ in Definition 3.7, we obtain

$$
H(\boldsymbol{A}, \boldsymbol{\alpha})= \begin{cases}\alpha_{1} \ln \rho\left(A_{1} A_{2}\right)+\left(\alpha_{2}-\alpha_{1}\right) \ln \rho\left(A_{2}\right) & \text { if } \alpha_{1} \leqslant \alpha_{2}, \\ \alpha_{2} \ln \rho\left(A_{1} A_{2}\right)+\left(\alpha_{1}-\alpha_{2}\right) \ln \rho\left(A_{1}\right) & \text { if } \alpha_{2} \leqslant \alpha_{1} .\end{cases}
$$

We are now ready to prove the following theorem.

Theorem 3.9. Let $\boldsymbol{u}$ be an infinite word with values taken from $\Sigma$ with frequency $\boldsymbol{\alpha}$ and suppose $\boldsymbol{u}$ generates $\left(q_{m}\right)$.

$$
\text { Then } \lim _{m \rightarrow \infty} \inf \left(\frac{1}{m}\right) \ln q_{m} \geqslant H(\boldsymbol{A}, \boldsymbol{\alpha}) \text {. }
$$

Proof. By Lemma 3.3 it suffices to show that

$$
\liminf _{\substack{m \rightarrow \infty \\ m \text { odd }}} \ln \operatorname{tr} W_{m}\left(A_{1}, A_{2}, \ldots, A_{n}\right) \geqslant H(\boldsymbol{A}, \boldsymbol{\alpha})
$$

We will prove this by induction on $n$.

If $n=1$, then in fact

$$
\lim _{m \rightarrow \infty}\left(\frac{1}{m}\right) \ln \operatorname{tr}\left(A_{1}^{m}\right)=\ln \rho\left(A_{1}\right)=H\left(A_{1}, 1\right),
$$

as can easily be checked by the reader ( $A_{1}$ is diagonalizable).

Assume now that $n \geqslant 2$ and that the result has been established for the case of $(n-1)$ arguments (in both $\boldsymbol{A}$ and $\boldsymbol{\alpha}$ ).

We will consider four possibilities for $\boldsymbol{\alpha} \in D_{n}$.

Case $1\left(\alpha_{1}<\alpha_{n}\right.$ (and so $\left.\alpha_{1}<\frac{1}{2}\right)$ ). Equation (3.1) is then applicable, and it follows by induction that

$$
\liminf _{\substack{m \rightarrow \infty \\ m \text { odd }}}\left(\frac{1}{m}\right) \ln \operatorname{tr} W_{m} \geqslant \alpha_{1} \ln \rho\left(A_{1}, A_{n}\right)+\left(1-2 \alpha_{1}\right) H(\boldsymbol{B}, \boldsymbol{\beta}),
$$

where, as before, $\boldsymbol{B}=\left(A_{2}, A_{3}, \ldots, A_{n}\right)$ and

$$
\boldsymbol{\beta}=\left(\frac{\alpha_{2}}{1-2 \alpha_{1}}, \ldots, \frac{\alpha_{n-1}}{1-2 \alpha_{1}}, \frac{\alpha_{n}-\alpha_{1}}{1-2 \alpha_{1}}\right) .
$$

But the right-hand side of $(3.4)$ is $H(\boldsymbol{A}, \boldsymbol{\alpha})$, by Definition 3.7, and so we have established the required inequality.

Case $2\left(\alpha_{n}<\alpha_{1}\right.$ (and so $\left.\left.\alpha_{n}<\frac{1}{2}\right)\right)$. This case is similar to Case (1). We proceed from Theorem 3.4 (b) and use $\boldsymbol{C}$ instead of $\boldsymbol{B}$.

Case $3\left(\boldsymbol{\alpha}_{\mathbf{1}}=\boldsymbol{\alpha}_{\boldsymbol{n}}<\frac{\mathbf{1}}{\mathbf{2}}\right)$. Let $I=\left\{m \in \mathbb{N}: m\right.$ odd and $\left.\alpha_{1}(m) \leqslant \alpha_{n}(m)\right\}$ and $J=\left\{m \in \mathbb{N}: m\right.$ odd and $\left.\alpha_{n}(m)<\alpha_{1}(m)\right\}$. At least one of $I$ or $J$ is infinite. If $I$ is infinite, then equation (3.1) gives

$$
\begin{aligned}
\liminf _{m \in I}\left(\frac{1}{m}\right) \ln \operatorname{tr} W_{m} & \geqslant \alpha_{1} \ln \rho\left(A_{1} A_{n}\right)+\left(1-2 \alpha_{1}\right) \liminf _{\substack{m \rightarrow \infty \\
m \text { odd }}}\left(\frac{1}{m^{*}}\right) \ln \operatorname{tr} W_{m^{*}} \\
& \geqslant H(\boldsymbol{A}, \boldsymbol{\alpha})
\end{aligned}
$$


by the inductive hypothesis. If also $J$ is infinite, we use $\alpha_{n}$ instead and get

$$
\liminf _{m \in J}\left(\frac{1}{m}\right) \ln \operatorname{tr} W_{m} \geqslant H(\boldsymbol{A}, \boldsymbol{\alpha}),
$$

and hence

$$
\liminf _{\substack{m \rightarrow \infty \\ m \text { odd }}}\left(\frac{1}{m}\right) \ln \operatorname{tr} W_{m} \geqslant H(\boldsymbol{A}, \boldsymbol{\alpha})
$$

If one of $I, J$ is finite, then we just have one liminf to consider.

Case $4\left(\boldsymbol{\alpha}_{\mathbf{1}}=\boldsymbol{\alpha}_{\boldsymbol{n}}=\frac{\mathbf{1}}{2}\right)$. Let $\epsilon>0$. Then for $m \geqslant m_{0}(\epsilon)$ we have that

$$
\frac{\alpha_{1}(m)}{m}>\frac{1}{2}-\epsilon \text { and } \quad \frac{\alpha_{n}(m)}{m}>\frac{1}{2}-\epsilon .
$$

By Theorem 3.4, where we replace the final trace by 1 , we easily have $\operatorname{tr} W_{m} \geqslant$ $\rho\left(A_{1} A_{n}\right)^{(m / 2)-\epsilon m}$ and hence

$$
\liminf _{\substack{m \rightarrow \infty \\ m \text { odd }}}\left(\frac{1}{m}\right) \ln \operatorname{tr} W_{m} \geqslant \frac{1}{2} \ln \rho\left(A_{1} A_{n}\right)=H\left(\boldsymbol{A}, \boldsymbol{f}_{1 n}\right),
$$

which is the required bound.

Let us now set $F(\boldsymbol{A}, \boldsymbol{\alpha})=(M(\boldsymbol{A}, \boldsymbol{\alpha}) / H(\boldsymbol{A}, \boldsymbol{\alpha}))$. Then we have the following corollary.

Corollary 3.10. $L(\boldsymbol{u}) \leqslant F(\boldsymbol{A}, \boldsymbol{\alpha})$.

Proof. Clear.

Theorem 3.11.

$$
L(\boldsymbol{u}) \leqslant \max _{1 \leqslant i<j \leqslant n}\left\{\frac{\ln \rho\left(A_{i}\right) \rho\left(A_{j}\right)}{\ln \rho\left(A_{i} A_{j}\right)}\right\} .
$$

Proof. $F(\boldsymbol{A}, \boldsymbol{\alpha})$ is the piecewise quotient of two linear functions (in $\boldsymbol{\alpha}$ ) and hence attains its maximum at a vertex of one of the defining $\Delta_{b}$ simplexes. By Proposition 3.6 the vertices of $\Delta_{\boldsymbol{b}}$ are elements of $S_{n}=\left\{\boldsymbol{f}_{i j}, 1 \leqslant i<j \leqslant n\right\} \bigcup\left\{\boldsymbol{e}_{i}: 1 \leqslant i \leqslant n\right\}$. We then compute

$$
\begin{aligned}
F\left(\boldsymbol{A}, \boldsymbol{e}_{i}\right) & =\frac{\alpha_{i} \ln \rho\left(A_{i}\right)}{H\left(\boldsymbol{\alpha}, \boldsymbol{e}_{i}\right)}=\frac{\alpha_{i} \ln \rho\left(A_{i}\right)}{\alpha_{i} \ln \rho\left(A_{i}\right)}=1 \\
F\left(\boldsymbol{A}, \boldsymbol{f}_{i j}\right) & =\frac{\frac{1}{2} \ln \rho\left(A_{i}\right)+\frac{1}{2} \ln \rho\left(A_{j}\right)}{H\left(\boldsymbol{A}, \boldsymbol{f}_{i j}\right)}=\frac{\ln \rho\left(A_{i}\right) \rho\left(A_{j}\right)}{\ln \rho\left(A_{i} A_{j}\right)},
\end{aligned}
$$

and so deduce that

$$
\max _{\boldsymbol{\alpha} \in S_{n}} F(\boldsymbol{A}, \boldsymbol{\alpha})=\max _{1 \leqslant i<j \leqslant n} \frac{\ln \rho\left(A_{i}\right) \rho\left(A_{j}\right)}{\ln \rho\left(A_{i} A_{j}\right)} .
$$

Hence, by Corollary 3.10, the result follows. 
In $[\mathbf{1}]$ it was shown that

$$
\max _{1 \leqslant a_{1}<a_{2}}\left(\frac{\ln \rho\left(A_{1}\right) \rho\left(A_{2}\right)}{\ln \rho\left(A_{1} A_{2}\right)}\right)<1.129
$$

and that the maximum is attained when $a_{1}=1, a_{2}=13$. Thus it follows from Theorem 3.11 that $L(\boldsymbol{u})<1.129$ for all infinite words with frequency and whose values come from a finite set of positive integers. For $n \geqslant 3$, we can make a slight improvement on the estimate for $L(\boldsymbol{u})$ if we can also assume that all symbols occur with the same frequency. We discuss this in our next section.

\section{Infinite words with uniform distribution}

If $|\Sigma|=n$ and the infinite word $\boldsymbol{u}$ has values from $\Sigma$ with frequency $\alpha_{i}=(1 / n)$ for $1 \leqslant i \leqslant n$, then we say that $\boldsymbol{u}$ is a word with uniform distribution.

If we set

$$
\boldsymbol{g}_{n}=\left(\frac{1}{n}, \frac{1}{n}, \ldots, \frac{1}{n}\right) \in D_{n}
$$

then from their respective definitions, we have

$$
M\left(\boldsymbol{A}, \boldsymbol{g}_{n}\right)=\frac{1}{n} \sum_{i=1}^{n} \ln \rho\left(A_{i}\right)
$$

and

$$
H\left(\boldsymbol{A}, \boldsymbol{g}_{n}\right)= \begin{cases}\frac{1}{n} \sum_{i=1}^{\lfloor n / 2\rfloor} \ln \rho\left(A_{i} A_{n+1-i}\right) & \text { if } n \text { is even, } \\ \frac{1}{n}\left\{\sum_{i=1}^{\lfloor n / 2\rfloor} \ln \rho\left(A_{i} A_{n+1-i}\right)+\ln \rho\left(A_{\lceil n / 2\rceil}\right)\right\} & \text { if } n \text { is odd. }\end{cases}
$$

For $1 \leqslant x \leqslant y$, let

$$
X=\left[\begin{array}{ll}
x & 1 \\
1 & 0
\end{array}\right], \quad Y=\left[\begin{array}{ll}
y & 1 \\
1 & 0
\end{array}\right]
$$

and consider the function $l(x, y)=1.1 \ln \rho(X Y)-\ln \rho(X) \rho(Y)$. Then

$$
l(x, y)=1.1 \ln \left(\frac{1}{2}\left(x y+2+\sqrt{x^{2} y^{2}+4 x y}\right)\right)-\ln \left(\frac{1}{4}\left(x+\sqrt{x^{2}+4}\right)\left(y+\sqrt{y^{2}+4}\right)\right) .
$$

The function $l$ has various properties, summarized in the following lemma.

Lemma 4.1. The function $l(x, y)$ satisfies the following conditions.

(a) If $1 \leqslant x_{1} \leqslant x_{2} \leqslant y$, then $l\left(x_{1}, y\right) \leqslant l\left(x_{2}, y\right)$.

(b) If $2 \leqslant x \leqslant y_{1} \leqslant y_{2}$, then $l\left(x, y_{1}\right) \leqslant l\left(x, y_{2}\right)$. 
(c) $\min _{y \geqslant 2} l(1, y)>-0.083$.

(d) $\min _{y \geqslant 3} l(2, y)=l(2,3)(>0.19)$.

Proof. Omitted.

Proposition 4.2. If $n \geqslant 3$, then $F\left(\boldsymbol{A}, \boldsymbol{g}_{n}\right)<1.1$.

\section{Proof.}

(1) $n=3$. Then

$$
F\left(\boldsymbol{A}, \boldsymbol{g}_{3}\right)=\frac{\ln \rho\left(A_{1}\right) \rho\left(A_{3}\right)+\ln \rho\left(A_{2}\right)}{\ln \rho\left(A_{1} A_{3}\right)+\ln \rho\left(A_{2}\right)} .
$$

So $F\left(\boldsymbol{A}, \boldsymbol{g}_{3}\right)<1.1$ is equivalent to $l\left(a_{1}, a_{3}\right)+0.1 \ln \rho\left(A_{2}\right)>0$. From parts (b) and (d) of Lemma 4.1 we see that $l\left(a_{1}, a_{3}\right)>0$ if $a_{1} \geqslant 2$. If $a_{1}=1$, then $a_{2} \geqslant 2$ so that $\rho\left(A_{2}\right) \geqslant 1+\sqrt{2}$ and thus

$$
\begin{aligned}
l\left(a_{1}, a_{3}\right)+0.1 \ln \rho\left(A_{2}\right) & \geqslant \min _{y \geqslant 2} l(1, y)+0.1 \ln (1+\sqrt{2}) \\
& >-0.083+0.088=0.05>0 .
\end{aligned}
$$

Thus in either case we have established that $F\left(\boldsymbol{A}, \boldsymbol{g}_{3}\right)<1.1$.

(2) $n$ odd, $n \geqslant 5$. Then $F\left(\boldsymbol{A}, \boldsymbol{g}_{n}\right)<1.1$ if and only if

$$
\sum_{l=1}^{\lfloor n / 2\rfloor} l\left(a_{i}, a_{n+1-i}\right)+0.1 \ln \rho\left(A_{\lceil n / 2\rceil}\right)>0 .
$$

The left-hand side of $(4.1)$ is at least $l\left(a_{1}, a_{n}\right)+0.1 \ln \rho\left(A_{2}\right)$ and hence is greater than 0 by part (1).

(3) $n$ even, $n \geqslant 4$. Then

$$
F\left(\boldsymbol{A}, \boldsymbol{g}_{n}\right)<1.1 \Leftrightarrow \sum_{i=1}^{\lfloor n / 2\rfloor} l\left(a_{i}, a_{n+1-i}\right)>0 .
$$

We will show that $l\left(a_{1}, a_{n}\right)+l\left(a_{2}, a_{n-1}\right)>0$.

By Lemma 4.1,

$$
\begin{aligned}
l\left(a_{1}, a_{n}\right)+l\left(a_{2}, a_{n-1}\right) & \geqslant \min _{y \geqslant 3} l(1, y)+\min _{y \geqslant 3} l(2, y) \\
& >-0.083+0.19>0 .
\end{aligned}
$$

Corollary 4.3. If $\boldsymbol{u}$ is an infinite word taking values in the finite set $\Sigma$ with uniform distribution and $|\Sigma| \geqslant 3$, then $L(\boldsymbol{u})<1.1$.

It is possible to show that $L(\boldsymbol{u})<1.09$ if $|\Sigma| \geqslant 4$, and presumably we would get smaller bounds as $|\Sigma| \rightarrow \infty$. 


\section{Expansion factors for $\{\lfloor m \theta\rfloor \bmod n\}$}

Suppose $\theta$ is irrational with $0<\theta<1$ and $n \geqslant 2$. We set $w_{m}=\lfloor m \theta\rfloor \bmod n$ and $\boldsymbol{w}=w_{1} w_{2} \ldots$ If $\Phi:\{0,1, \ldots, n-1\} \rightarrow \Sigma$ is a bijection, then the infinite word $\boldsymbol{u}$ is defined by setting $u_{m}=\Phi\left(w_{m}\right)$ for $m \geqslant 1$. $\boldsymbol{u}$ is said to be derived from $\boldsymbol{w}$ and we also write $\boldsymbol{u}=\Phi \circ \boldsymbol{w}$. From Definition 1.1 it is evident that $\boldsymbol{u}$ has a segment expansion factor greater than or equal to $\gamma$ if and only if $\boldsymbol{w}$ has a segment expansion factor greater than or equal to $\gamma$.

It will turn out that, in order to obtain the transcendence of continued fractions associated with such $\boldsymbol{u}$, we need to obtain conditions on $\theta$ to guarantee that $\boldsymbol{w}$ will have a segment expansion factor greater that $\frac{3}{2}$.

Let the continued fraction of $\theta$ be $\left[0, b_{1}, b_{2}, \ldots\right]$ with convergents $\left(P_{k} / Q_{k}\right)_{k \geqslant 0}$. If $t$ is a non-negative integer, then we set

$$
P_{k, t}=t P_{k+1}+P_{k}, \quad Q_{k, t}=t Q_{k+1}+Q_{k} .
$$

Thus, in particular,

$$
P_{k, 0}=P_{k}, \quad P_{k, b_{k+2}}=P_{k+2}
$$

and

$$
Q_{k, 0}=Q_{k}, \quad Q_{k, b_{k+2}}=Q_{k+2} .
$$

If $b_{k+2} \geqslant 2$ and $1 \leqslant t \leqslant b_{k+2}-1$, then $P_{k, t} / Q_{k, t}$ is called a median convergent to $\theta$ (see [8] for further information).

The following proposition generalizes what was proved in [3].

Proposition 5.1. Suppose $k \geqslant 0$ and $0 \leqslant t \leqslant b_{k+2}-1$. Then for all integers $m$ satisfying $1 \leqslant m<Q_{k, t+1}$ we have

$$
\lfloor m \theta\rfloor= \begin{cases}\left\lfloor m \frac{P_{k, t}}{Q_{k, t}}\right\rfloor & \text { if } k \text { is even, } \\ \left\lceil m \frac{P_{k, t}}{Q_{k, t}}\right\rceil-1 & \text { if } k \text { is odd. }\end{cases}
$$

\section{Proof.}

(1) First consider when $k$ is even. From the classical inequalities

$$
0<Q_{k+2} \theta-P_{k+2}<Q_{k+2}^{-1}
$$

we derive

$$
\lfloor m \theta\rfloor=\left\lfloor m \frac{P_{k+2}}{Q_{k+2}}\right\rfloor \quad \text { for } 1 \leqslant m \leqslant Q_{k+2} .
$$

We now show that if $t$ satisfies $0 \leqslant t \leqslant b_{k+2}-1$, then

$$
\left\lfloor m \frac{P_{k, t}}{Q_{k, t}}\right\rfloor=\left\lfloor m \frac{P_{k, t+1}}{Q_{k, t+1}}\right\rfloor \text { for } 1 \leqslant m<Q_{k, t+1} .
$$


From the basic theory we have

$$
\frac{P_{k, t+1}}{Q_{k, t+1}}-\frac{P_{k, t}}{Q_{k, t}}=\frac{1}{Q_{k, t+1} Q_{k, t}},
$$

and therefore if $1 \leqslant m<Q_{k, t+1}$, we obtain

$$
m \frac{P_{k, t}}{Q_{k, t}}<m \frac{P_{k, t+1}}{Q_{k, t+1}}<m \frac{P_{k, t}}{Q_{k, t}}+\frac{1}{Q_{k, t}},
$$

from which (5.2) follows immediately.

If we put $t=b_{k+2}-1$ in (5.2) and use (5.1), we find that $\lfloor m \theta\rfloor=\left\lfloor m\left(P_{k, t} / Q_{k, t}\right)\right\rfloor$ for $1 \leqslant m<Q_{k, t+1}$. We can then put $t=b_{k+2}-2$ in (5.2) and see that the required result holds. Continuing in this manner we establish the result for all $t: 0 \leqslant t \leqslant b_{k+2}-1$.

(2) Now consider the case when $k$ is odd. We can similarly show that if $1 \leqslant m \leqslant Q_{k+2}$, then

$$
\lfloor m \theta\rfloor=\left\lceil m \frac{P_{k+2}}{Q_{k+2}}\right\rceil-1,
$$

and if $1 \leqslant m<Q_{k, t+1}, 0 \leqslant t \leqslant b_{k+2}-1$, then

$$
\left\lceil m \frac{P_{k, t}}{Q_{k, t}}\right\rceil=\left\lceil m \frac{P_{k, t+1}}{Q_{k, t+1}}\right\rceil
$$

and the result follows as before.

Corollary 5.2. Suppose $k \geqslant 0$ and $0 \leqslant t \leqslant b_{k+2}-1$. Then $\left\lfloor\left(Q_{k, t}+r\right) \theta\right\rfloor=P_{k, t}+\lfloor r \theta\rfloor$ for $1 \leqslant r \leqslant Q_{k+1}-1$.

Proof. Omitted.

For each $k \geqslant 0$ and for $0 \leqslant t \leqslant b_{k+2}-1$ we define the following prefixes of $\boldsymbol{w}$ :

$$
\begin{aligned}
X_{k, t} & =\{\lfloor m \theta\rfloor \bmod n\}_{1 \leqslant m \leqslant Q_{k, t}}, \\
Z_{k, t} & =\{\lfloor m \theta\rfloor \bmod n\}_{1 \leqslant m<Q_{k, t+1}} .
\end{aligned}
$$

For convenience we write $X_{k}=X_{k, 0}$ and $Z_{k}=Z_{k, 0}$. The prefix partial order will be denoted by ' $\leqslant$ ', so it is evident that $X_{k, t} \leqslant Z_{k, t}$.

We are now able to prove the following proposition.

Proposition 5.3. Suppose $\theta=\left[0, b_{1}, b_{2}, \ldots\right]$ with convergents $\left\{P_{k} / Q_{k}\right\}_{k \geqslant 0}$ and $\boldsymbol{w}=\{\lfloor m \theta\rfloor \bmod n\}_{m \geqslant 1}$.

(a) If there is an infinite number of $k$ with $P_{k} \equiv 0 \bmod n$, then $\boldsymbol{w}$ has a prefix expansion factor greater than or equal to 2 . 
(b) Suppose $M=\lim \sup _{r \rightarrow \infty} b_{r}<\infty$. If there is an infinite number of $k$ with $P_{k}+P_{k+1} \equiv 0 \bmod n$ and $b_{k+2} \geqslant 2$, then $\boldsymbol{w}$ has a prefix expansion factor greater than or equal to $\frac{3}{2}+1 /(8 M)$.

(c) If there is an infinite number of $k$ with $b_{k+1} \geqslant \frac{3}{2} n$, then $\boldsymbol{w}$ has a prefix expansion factor greater than or equal to $\frac{3}{2}+1 / n$.

\section{Proof.}

(a) Suppose $P_{k} \equiv 0 \bmod n$. By Corollary $5.2\left(\right.$ with $t=0$ ), we can write $Z_{k}=X_{k} Y_{k}$ with $Y_{k} \leqslant X_{k}^{s}$ for some integer $s$. Let $U_{k}=\lambda, V_{k}=X_{k}, W_{k}=Y_{k}$. Then

$$
\frac{\left|V_{k} W_{k}\right|}{\left|V_{k}\right|}=\frac{Q_{k+1}+Q_{k}-1}{Q_{k}}>2 .
$$

Since there are an infinite number of such $k$, we can conclude that $\boldsymbol{w}$ has a prefix expansion factor greater than or equal to 2 .

(b) Suppose $P_{k}+P_{k+1} \equiv 0 \bmod n$ and $b_{k+2} \geqslant 2$. Applying Corollary 5.2 with $t=1$ gives $\left\lfloor\left(Q_{k, 1}+r\right) \theta\right\rfloor \equiv\lfloor r \theta\rfloor \bmod n$ for $1 \leqslant r \leqslant Q_{k+1}-1$. Hence $Z_{k, 1}=X_{k, 1} Y_{k, 1}$ with $Y_{k, 1} \leqslant X_{k, 1}^{s}$ for some positive integer $s$. Set $U_{k}=\lambda, V_{k}=X_{k, 1}, W_{k}=Y_{k, 1}$ and we find that

$$
\frac{\left|V_{k} W_{k}\right|}{\left|V_{k}\right|}=\frac{2 Q_{k+1}+Q_{k}-1}{Q_{k+1}+Q_{k}} .
$$

The assumption that $\lim \sup _{r \rightarrow \infty} b_{r}=M \geqslant 2$ gives, for all sufficiently large $k$,

$$
\frac{Q_{k+1}}{Q_{k}} \geqslant 1+\frac{1}{M+1}
$$

and hence that

$$
\frac{\left|V_{k} W_{k}\right|}{\left|V_{k}\right|} \geqslant \frac{3}{2}+\frac{1}{8 M}
$$

(c) Suppose $b_{k+1} \geqslant \frac{3}{2} n$. Put $\tilde{X}_{k}=\{\lfloor m \theta\rfloor \bmod n\}_{1 \leqslant m \leqslant n Q_{k}}$. Then $\left|Z_{k}\right|=Q_{k+1}+Q_{k}-$ $1>n Q_{k}=\left|\tilde{X}_{k}\right|$ so $\tilde{X}_{k} \leqslant Z_{k}$. Thus $Z_{k}=\tilde{X}_{k} \tilde{Y}_{k}$ and $\tilde{Y}_{k} \leqslant \tilde{X}_{k}^{s}$ for some positive integer $s$. Setting $U_{k}=\lambda, V_{k}=\tilde{X}_{k}, W_{k}=\tilde{Y}_{k}$ we obtain

$$
\frac{\left|V_{k} W_{k}\right|}{\left|V_{k}\right|}=\frac{Q_{k+1}+Q_{k}-1}{n Q_{k}} \geqslant \frac{\frac{3}{2} n Q_{k}+Q_{k-1}+Q_{k}-1}{n Q_{k}} \geqslant \frac{3}{2}+\frac{1}{n} .
$$

\section{Remark 5.4.}

(1) Proposition 5.3 only refers to results involving $t=0$ and $t=1$. In fact if we consider $t \geqslant 2$ (so that necessarily $b_{k+2} \geqslant 3$ ) we find that

$$
\frac{\left|Z_{k, t}\right|}{\left|X_{k, t}\right|}=\frac{Q_{k, t+1}-1}{Q_{k, t}}<\frac{3}{2}
$$

and we do not have an instance to demonstrate that $\boldsymbol{w}$ has a prefix expansion factor greater than or equal to $\frac{3}{2}$. 
(2) It is possible to obtain analogous results to Proposition 5.3 when $\theta>1$, but we have suppressed the details.

We now conclude this section by finding a situation where $\boldsymbol{w}$ has a segment expansion factor greater than $\frac{3}{2}$. From Corollary 5.2 (with $t=0, k \geqslant 0$ ) we see that

$$
\left\lfloor\left(Q_{k+1}+r\right) \theta\right\rfloor=\left\lfloor\left(Q_{k}+r\right) \theta\right\rfloor+P_{k+1}-P_{k} \quad \text { for } 1 \leqslant r \leqslant Q_{k+1}-1,
$$

and we can now prove the following proposition.

Proposition 5.5. Suppose $M=\lim \sup _{r \rightarrow \infty} b_{r}<\infty$. If there are an infinite number of $k$ satisfying $P_{k} \equiv P_{k+1} \bmod n$ and $b_{k+1} \geqslant 3$, then $\boldsymbol{w}$ has a segment expansion factor greater than or equal to $\frac{3}{2}+1 /(12 M)$.

Proof. Assume $P_{k} \equiv P_{k+1} \bmod n$ and let $U_{k}=\{\lfloor m \theta\rfloor \bmod n\}_{1 \leqslant m \leqslant Q_{k}}$,

$$
V_{k}=\{\lfloor m \theta\rfloor \bmod n\}_{Q_{k}<m \leqslant Q_{k+1}}, \quad W_{k}=\{\lfloor m \theta\rfloor \bmod n\}_{Q_{k+1}<m<2 Q_{k+1}} .
$$

Then $U_{k} V_{k} W_{k} \leqslant \boldsymbol{w}$. By the observation just preceding Proposition 5.5 it is evident that $W_{k} \leqslant V_{k}^{s}$ for some positive integers $s$. Also

$$
\frac{\left|U_{k} V_{k} W_{k}\right|}{\left|U_{k}\right|+\left|U_{k} V_{k}\right|}=\frac{2 Q_{k+1}-1}{Q_{k}+Q_{k+1}} \geqslant \frac{3}{2}+\frac{1}{12 M},
$$

since under our present assumption

$$
\frac{Q_{k+1}}{Q_{k}} \geqslant 3+\frac{1}{M+1}
$$

for large $k$ instances.

\section{Approximation by quadratic irrationals}

We now proceed to make the connection between segment/prefix expansion factors and quadratic approximation. If $\eta$ is a quadratic irrational satisfying the minimal equation $a \eta^{2}+b \eta+c=0$, where $a, b, c \in \mathbb{Z}$ with $\operatorname{gcd}(a, b, c)=1$, then we set $H(\eta)=$ $\max \{|a|,|b|,|c|\}$. As before, $\boldsymbol{u}=u_{1} u_{2} \ldots, \alpha=\left[0, u_{1}, u_{2}, \ldots\right]$ and $\left\{p_{m} / q_{m}\right\}_{m \geqslant 0}$ is the sequence of convergents of $\alpha$.

The following result is a refinement of the estimate given by Baker in [2].

Lemma 6.1. Let

$$
\eta=\left[0, u_{1}, u_{2}, \ldots, u_{h-1}, \overline{u_{h}, \ldots, u_{h+k-1}}\right] .
$$

Then $H(\eta)<2 q_{h-1} q_{h+k-1}$.

Proof. Let $\eta_{h}=\left[\overline{u_{h}, u_{h+1}, \ldots, u_{h+k-1}}\right]$. Then

$$
\eta=\frac{p_{h-1} \eta_{h}+p_{h-2}}{q_{h-1} \eta_{h}+q_{h-2}}=\frac{p_{h+k-1} \eta_{h}+p_{h+k-2}}{q_{h+k-1} \eta_{h}+q_{h+k-2}} .
$$


Eliminating $\eta_{h}$, we obtain $P \eta^{2}+Q \eta+R=0$, where

$$
\begin{aligned}
& P=q_{h-2} q_{h+k-1}-q_{h-1} q_{h+k-2}, \\
& Q=q_{h-1} p_{h+k-2}+p_{h-1} q_{h+k-2}-p_{h-2} q_{h+k-1}-q_{h-2} p_{h+k-1}, \\
& R=p_{h-2} p_{h+k-1}-p_{h-1} p_{h+k-2} .
\end{aligned}
$$

Now $0<\eta<1$ so $p_{r} \leqslant q_{r}$ for $r \geqslant 0$. Hence

$$
|P| \leqslant q_{h-1} q_{h+k-1}, \quad|R| \leqslant q_{h-1} q_{h+k-1}
$$

and

$$
|Q| \leqslant \max \left\{2 q_{h-1} q_{h+k-2}, 2 q_{h-2} q_{h+k-1}\right\} \leqslant 2 q_{h-1} q_{h+k-1} .
$$

Lemma 6.2. Suppose $\left\{U_{k}\right\}_{k \geqslant 1}$ and $\left\{V_{k}\right\}_{k \geqslant 1}$ are two families of words (in $\Sigma$ ) satisfying the two conditions:

(i) $U_{k} V_{k}<\boldsymbol{u}$; and

(ii) $\lim _{k \rightarrow \infty}\left|V_{k}\right|=\infty$.

Then

$$
\limsup _{k \rightarrow \infty}\left(\frac{\ln q_{\left|U_{k}\right|} q_{\left|U_{k} V_{k}\right|}}{\left|U_{k}\right|+\left|U_{k} V_{k}\right|}\right) \leqslant \limsup _{m \rightarrow \infty}\left(\frac{1}{m}\right) \ln q_{m} .
$$

Proof. Let $M=\lim \sup _{m \rightarrow \infty}(1 / m) \ln q_{m}$ and let $\epsilon>0$. Then there exists $m_{0} \geqslant 1$ such that if $m \geqslant m_{0}$, then $(1 / m) \ln q_{m}<M+\frac{1}{2} \epsilon$. Let $A=\max _{0 \leqslant m \leqslant m_{0}-1} \ln q_{m}$. Since $\lim _{k \rightarrow \infty}\left|V_{k}\right|=\infty$, then there exists $k_{0}$ such that if $k \geqslant k_{0}$, then $\left|V_{k}\right| \geqslant m_{0}$ and $A<$ $\frac{1}{2} \epsilon\left|V_{k}\right|$. Now put $I=\left\{k:\left|U_{k}\right|<m_{0}\right\}$ and $J=\left\{k:\left|U_{k}\right| \geqslant m_{0}\right\}$. If $k \in I, k \geqslant k_{0}$ we have

$$
\frac{\ln q_{\left|U_{k}\right|} q_{\left|U_{k} V_{k}\right|}}{\left|U_{k}\right|+\left|U_{k} V_{k}\right|} \leqslant \frac{A+\ln q_{\left|U_{k}\right| V_{k} \mid}}{\left|U_{k} V_{k}\right|}<\frac{1}{2} \epsilon+M+\frac{1}{2} \epsilon=M+\epsilon,
$$

whereas, if $k \in J, k \geqslant k_{0}$ we have

$$
\begin{aligned}
\frac{\ln q_{\left|U_{k}\right|} q_{\left|U_{k} V_{k}\right|}}{\left|U_{k}\right|+\left|U_{k} V_{k}\right|} & =\frac{\left|U_{k}\right|}{\left|U_{k}\right|+\left|U_{k} V_{k}\right|} \frac{\ln q_{\left|U_{k}\right|}}{\left|U_{k}\right|}+\frac{\left|U_{k} V_{k}\right|}{\left|U_{k}\right|+\left|U_{k} V_{k}\right|} \frac{\ln q_{\left|U_{k} V_{k}\right|}}{\left|U_{k} V_{k}\right|} \\
& <M+\frac{1}{2} \epsilon,
\end{aligned}
$$

since it is a convex combination of two numbers, both less than $M+\frac{1}{2} \epsilon$.

The following theorem generalizes Theorem 4 of [1].

Theorem 6.3. If $\alpha$ is not a quadratic irrational (that is, $\boldsymbol{u}$ is not ultimately periodic) and if $\boldsymbol{u}$ has a segment expansion factor greater than or equal to $\gamma$, then there is a sequence of quadratic irrationals $\left(\alpha_{k}\right)$ which satisfies

$$
\left|\alpha-\alpha_{k}\right|<\frac{1}{H\left(\alpha_{k}\right)^{2 \gamma / L(\alpha)}},
$$

where $H\left(\alpha_{k}\right)$ denotes the height of $\alpha_{k}$. 
Proof. By assumption there are three families of words $\left\{U_{k}\right\}_{k \geqslant 1},\left\{V_{k}\right\}_{k \geqslant 1},\left\{W_{k}\right\}_{k \geqslant 1}$ satisfying the requirements of Definition 1.1. Set

$$
\alpha_{k}=\left[0, u_{1}, u_{2}, \ldots, u_{\left|U_{k}\right|}, \overline{u_{\left|U_{k}\right|+1}, \ldots, u_{\left|U_{k} V_{k}\right|}}\right] .
$$

By standard theory, $\alpha_{k}$ is a quadratic irrational. Furthermore, since $\lim _{k \rightarrow \infty}\left|V_{k}\right|=$ $\infty$, it is evident that there an infinite number of distinct $\alpha_{k}$ s. By Lemma 6.1, $H\left(\alpha_{k}\right)<2 q_{\left|U_{k}\right|} q_{\left|U_{k} V_{k}\right|}$. In addition, from Definition 1.1 it is clear that $\alpha$ and $\alpha_{k}$ have the same first $\left|U_{k} V_{k} W_{k}\right|$ partial quotients. Thus

$$
\left|\alpha-\alpha_{k}\right| \leqslant \frac{1}{q_{\left|U_{k} V_{k} W_{k}\right|}^{2}} .
$$

The required result will then follow if we can show that

$$
q_{\left|U_{k} V_{k} W_{k}\right|}^{2} \geqslant\left(2 q_{\left|U_{k}\right|} q_{\left|U_{k} V_{k}\right|}\right)^{2 \gamma / L(\alpha)}
$$

for all $k$ that are large enough. Using Lemma 6.2,

$$
\begin{aligned}
\liminf _{k \rightarrow \infty} \frac{2 \ln q_{\left|U_{k} V_{k} W_{k}\right|}}{\ln \left(2 q_{\left|U_{k}\right|} q_{\left|U_{k} V_{k}\right|}\right)} & =2 \liminf _{k \rightarrow \infty}\left\{\frac{\ln q_{\left|U_{k} V_{k} W_{k}\right|}}{\ln \left(q_{\left|U_{k}\right|} \mid q_{\left|U_{k} V_{k}\right|}\right)}\right\} \\
& \geqslant 2 \liminf _{k \rightarrow \infty}\left\{\left(\frac{\ln q_{\left|U_{k} V_{k} W_{k}\right|}}{\left|U_{k} V_{k} W_{k}\right|} / \frac{\ln \left(q_{\left|U_{k}\right|} \mid q_{\left|U_{k} V_{k}\right|}\right)}{\left|U_{k}\right|+\left|U_{k} V_{k}\right|}\right) \frac{\left|U_{k} V_{k} W_{k}\right|}{\left|U_{k}\right|+\left|U_{k} V_{k}\right|}\right\} \\
& \geqslant 2 \frac{\liminf _{m \rightarrow \infty}(1 / m) \ln q_{m}}{\limsup \sup _{m \rightarrow \infty}(1 / m) \ln q_{m}} \cdot \gamma \\
& =\frac{2 \gamma}{L(\boldsymbol{\alpha})},
\end{aligned}
$$

as required.

Theorem 6.4. Let $0<\theta<1$ be irrational with continued fraction expansion $\theta=$ $\left[0, b_{1}, b_{2}, \ldots\right]$ and principal convergents $\left(P_{k} / Q_{k}\right)_{k \geqslant 0}$. Let $n$ be an integer greater than or equal to 2 and $\Sigma \subset \mathbb{Z}^{+}$with $|\Sigma|=n$. Suppose $\Phi:\{0,1, \ldots, n-1\} \rightarrow \Sigma$ is a bijection, $\boldsymbol{w}=\{\lfloor m \theta\rfloor \bmod n\}_{m \geqslant 1}$ and $\boldsymbol{u}=\Phi \circ \boldsymbol{w}$ with associated continued fraction $\alpha$.

Then $\alpha$ is transcendental if any of the following conditions hold for an infinite number of positive integers $k$ :

(a) $P_{k} \equiv 0 \bmod n$;

(b) $b_{k+1} \geqslant \frac{3}{2} n$;

(c) $P_{k}+P_{k+1} \equiv 0 \bmod n$;

(d) $P_{k} \equiv P_{k+1} \bmod n$.

Proof. Since $\theta$ is irrational it is clear that $\alpha$ is neither a rational nor a quadratic irrational. In view of Theorem 6.3 and Schmidt's Theorem [9], the transcendence of $\alpha$ will be proved provided $(2 \gamma / L(\alpha))>3+\delta$, for some $\delta>0$. For any irrational $\theta$, it can 
be shown by classical ergodic theory that $L(\boldsymbol{u})=1$ (cf. $[\mathbf{5}, \mathbf{7}]$ ). (I thank the referee for bringing this to my attention.) A purely elementary proof of this fact can be found in [4]. Thus we need only demonstrate that $\boldsymbol{w}$ has a segment expansion factor $\gamma \geqslant \frac{3}{2}+\frac{1}{2} \delta$ for some $\delta>0$.

If (a) or (b) holds for an infinite number of $k$, then Proposition 5.3 (a), (c) gives the required result. Suppose that (c) holds, but neither (a) nor (b) holds for an infinite number of $k$. Then $\limsup _{r \rightarrow \infty} b_{r} \leqslant \frac{3}{2} n$ and $P_{r} \not \equiv 0 \bmod n$ for all sufficiently large $r$. Now if $b_{k+2}=1$, then $P_{k+2}=P_{k+1}+P_{k} \equiv 0 \bmod n$. So we must have $b_{k+2} \geqslant 2$ for all sufficiently large instances of (c). Proposition 5.3 (b) then yields the result.

Finally, suppose that (d) holds but none of (a)-(c) hold for an infinite number of $k$. It is then easy to check that we must have $b_{k+1} \geqslant 3$ for all sufficiently large instances of (d). We can then use Proposition 5.5 to complete the proof.

Theorem 6.4 immediately gives the result that $\alpha$ is always transcendental when $n$ equals 2 or 3 . If $n=5$ and $\theta=[0,1,3,1,1,1, \ldots]$, then for all $k \geqslant 1$, we find that none of the conditions (a)-(d) hold; so it is an open question whether the corresponding $\alpha$ can be shown to be transcendental by the methods of this paper.

Acknowledgements. I thank the referee for the many constructive comments and suggestions on how to improve this paper. Some of the work was done while visiting the University of Edinburgh and I express my gratitude for the hospitality shown to me.

\section{References}

1. J.-P. Allouche, J. L. Davison, M. Queffélec and L. Q. Zamboni, Transcendence of Sturmian or morphic continued fractions, J. Number Theory 91 (2001), 39-66.

2. A. BAKER, Continued fractions of transcendental numbers, Mathematika 9 (1962), 1-8.

3. J. L. DAvison, A class of transcendental numbers with bounded quotients, in Number Theory and Applications, Banff, AB, 1988, NATO Advanced Science Institutes Series C, Mathematical and Physical Sciences, no. 265 pp. 365-371 (Kluwer, Dordrecht, 1989).

4. J. L. DAvison, Levy constants for classes of continued fractions (unpublished).

5. C. Faivre, Distribution of Lévy constants for quadratic numbers, Acta Arithm. 61 (1992), 113-134.

6. G. HaRdy And E. Wright, An introduction to the theory of numbers (Oxford University Press, 1975).

7. H. Jager And P. Liardet, Distributions arithmétiques des dénominateurs de convergents de fractions continues, Nederl. Akad. Wetensch. Indag. Math. 50 (1988), 181-197.

8. S. LANG, An introduction to Diophantine approximation (Addison-Wesley, 1966).

9. W. Schmidt, On simultaneous approximation of two algebraic numbers by rationals, Acta Math. 119 (1967), 27-50. 